\title{
Relação entre os Estados Afetivos e as Teorias de Aprendizagem na Formação de Grupos em Ambientes CSCL
}

\author{
Rachel C. D. Reis ${ }^{1,2}$, Carla L. Rodriguez ${ }^{1,3}$, Geiser C. Challco ${ }^{1}$, Patrícia A. Jaques ${ }^{4}$, \\ Ig I. Bittencourt ${ }^{5}$, Seiji Isotani ${ }^{1}$ \\ ${ }^{1}$ Instituto de Ciências Matemáticas e de Computação - Universidade de São Paulo \\ (USP), Avenida Trabalhador São Carlense, 400 - Centro - 13566-590 - São Carlos, SP \\ ${ }^{2}$ Universidade Federal de Viçosa, campus Rio Paranaíba (UFV-CRP) \\ ${ }^{3}$ Instituto de Educação da Universidade de Lisboa (IE-ULisboa) \\ ${ }^{4}$ Universidade do Vale do Rio dos Sinos (UNISINOS) \\ ${ }^{5}$ Universidade Federal do Alagoas (UFAL) \\ rachel.reis@ufv.br, crpaiva@ie.ulisboa.pt, geiser@usp.br, \\ pjaques@unisinos.br, ig.ibert@gmail.com, sisotani@icmc.usp.br
}

\begin{abstract}
CSCL researchers have been investigating models based on learning theories to support the formation of high-performance learning groups. Despite of important contributions to this area, we observed the lack of studies employing affective states (e.g., personality trait) combined with other relevant elements in group formation (e.g., learning theories). Thus, this paper aims to present a proposal of relation between affective states and learner's roles based on the various collaborative learning theories. As results, we defined new learner's roles ("affective roles"), modeled using ontologies, to the CSCL context.
\end{abstract}

Resumo. Pesquisadores da CSCL têm investigado modelos baseados em teorias de aprendizagem para apoiar a formação de grupos de alta performance. Apesar das importantes contribuições nessa área, observou-se a falta de estudos que utilizem os estados afetivos (e.g., traços de personalidade) combinados a outros elementos importantes na formação de grupos (e.g., teorias de aprendizagem). Nesse sentido, este artigo tem como objetivo apresentar uma proposta de relação entre os estados afetivos e os papéis dos alunos, baseada nas diversas teorias de aprendizagem colaborativa. Como resultados, foram definidos novos papéis ("papéis afetivos"), modelados por meio de ontologias, para o contexto da CSCL.

\section{Introdução}

A Aprendizagem Colaborativa com Suporte Computacional (CSCL) é um campo de pesquisa multidisciplinar, caracterizado pelo uso de tecnologias para apoiar a interação e a colaboração entre os estudantes nas atividades em grupo e promover a construção do conhecimento individual e coletivo dos seus participantes [Tchounikine et al. 2010]. De acordo com Dillenbourg (2002) e Isotani et al. (2009), o sucesso dos cenários CSCL 
depende de um conjunto de fatores relacionados ao agrupamento e interação dos alunos, bem como na definição e condução das atividades em grupo.

Para que os benefícios da aprendizagem colaborativa sejam alcançados, é importante que na formação de grupos os alunos sejam organizados de forma que o processo de interação os remeta à argumentação, negociação, resolução de conflitos e compartilhamento de ideias [Inaba e Mizoguchi 2004; Roschelle e Teasley 1995]. Nesse sentido, alguns trabalhos científicos têm investigado o uso dos estados afetivos (e.g., traço de personalidade, emoção) na formação de grupos em ambientes CSCL [Lopes Filho et al. 2010; Hsu et al. 2008; Flor e Finger 2006; Quarto et al., 2006; Wang et al. 2007; Silva et al. 2009]. Apesar dos resultados positivos apresentados por esses trabalhos, pode-se observar que, em geral, os estados afetivos são utilizados de forma isolada, ou seja, sem a combinação com outros fatores importantes para a formação de grupos tais como, teorias e estratégias de aprendizagem adotadas, definição de papéis, padrões de interação, objetivos individuais, dentre outros [Isotani et al. 2009].

Dado que os benefícios educacionais da aprendizagem em ambientes CSCL estão fortemente relacionadas à definição de estratégias que orientem como os alunos são levados a interagir [Inaba e Mizoguchi, 2004] e, considerando a relevância dos estados afetivos na interação e construção do conhecimento dos alunos [Leite e Tassoni 2002], este trabalho tem como objetivo relacionar os estados afetivos aos papéis que os alunos podem assumir nas diversas teorias de aprendizagem colaborativa, para melhorar o planejamento instrucional de cenários CSCL, especificamente na formação de grupos de alta performance ${ }^{1}$. Como resultados, serão definidos novos papéis, que serão tratados nesse artigo como "papéis afetivos" (e.g. instrutor extrovertido), com novos comportamentos e requisitos (condições necessárias e desejadas) para o contexto da aprendizagem colaborativa. Essa nova configuração será modelada por meio de ontologias, como uma extensão à estrutura ontológica de papéis desenvolvida por Isotani et al. (2009).

Além dessa seção introdutória, este trabalho foi organizado da seguinte forma: na Seção 2 são apresentados alguns trabalhos relacionados, que utilizaram o estado afetivo traço de personalidade na formação de grupos de aprendizagem em ambientes CSCL. Na sequência, a Seção 3.1 discute as teorias de aprendizagem que dão suporte à aprendizagem colaborativa. Na Seção 3.2 tem-se a definição dos estados afetivos com foco na definição e caracterização dos traços de personalidade que são usados nesse trabalho. Na Seção 4 é feita a relação entre os estados afetivos e os papéis, mostrando sua representação por meio de um modelo de ontologias. Finalmente, na Seção 5 são apresentadas as conclusões, seguida das Referências utilizadas neste trabalho.

\footnotetext{
${ }^{1}$ Grupos de aprendizagem de alta performance, no contexto deste trabalho, referem-se a formação de grupos que promovam a interação de seus integrantes de forma que todos participem ativamente do processo de aprendizagem, colaborando para que os objetivos do trabalho sejam alcançados de forma plena.
} 


\section{Trabalhos Relacionados}

O processo de formação de grupos de alta performance é caracterizado como um dos desafios para o planejamento de cenários CSCL, principalmente no que se refere à definição dos fatores que devem ser considerados no agrupamento dos indivíduos [Isotani et al. 2009]. No que diz respeito ao uso do estado afetivo traço de personalidade na formação de grupos em ambientes CSCL, os trabalhos de Hsu et al. (2008), Flor e Finger (2006) e Quarto et al. (2006) investigam a composição dos grupos com base na heterogeneidade e/ou homogeneidade dos traços de personalidade. Como resultados, Flor e Finger (2006) afirmam que a diversidade dos grupos em termos de personalidade não influenciou na colaboração entre os alunos. Por outro lado, Quarto et al. (2006) concluem que grupos com características heterogêneas de personalidade favoreceram a interação e colaboração dos alunos, contrapondo Hsu et al. (2008) cuja pesquisa aponta que os grupos homogêneos apresentaram melhores resultados para determinadas tarefas.

Outros trabalhos apresentam o desenvolvimento de ferramentas que utilizam os traços de personalidade para formação automática de grupos de aprendizagem. Os resultados mostraram que os grupos formados automaticamente tiveram melhor desempenho que os grupos gerados aleatoriamente [Wang et al. 2007; Silva et al. 2010].

Vale observar que dentre os trabalhos apresentados nesta seção apenas o estudo de Silva et al. (2010) relacionou os traços de personalidade a outros fatores considerados na formação de grupos como, habilidades e papéis que os alunos podem desempenhar no grupo (e.g. implementer, resource investigator). Os demais trabalhos apresentados nesta seção utilizaram os traços de personalidade de forma isolada, ou seja, sem a combinação de outros fatores importantes para a formação de grupos.

\section{Metodologia}

Utilizou-se a metodologia de Engenharia de Ontologias, proposta por Mizoguchi (2004), para identificação de conceitos sobre traços de personalidade e suas relações com teorias de aprendizagem no contexto colaborativo. Para tanto, inicialmente são identificados os conceitos principais das teorias de aprendizagem em CSCL (Seção 3.1). Em seguida, são expostos os conceitos relacionados a traços de personalidade, extraídos e organizados durante a execução da pesquisa (Seção 3.2). E por fim, é feito o relacionamento entre estes conceitos de forma a formalizar o conceito de papéis afetivos em CSCL e seu impacto no processo de ensino-aprendizagem em grupo.

\subsection{Teorias de Aprendizagem e Ontologia de Aprendizagem Colaborativa}

Teorias de aprendizagem têm sido propostas por pesquisadores de diferentes áreas de conhecimento (e.g., Psicologia, Neurociência, etc) como uma tentativa de identificar e entender as condições essenciais para que o processo de aprendizagem ocorra de forma efetiva. $\mathrm{O}$ que normalmente acontece é que cada teoria determina o contexto em que a atividade de aprendizagem será realizada, o tipo de conhecimento e habilidade a ser desenvolvida, e o papel a ser desempenhado pelos aprendizes [Isotani et al. 2009]. 
Em ambientes CSCL, modelos baseados em teorias de aprendizagem têm sido desenvolvidos e avaliados por diversos pesquisadores [Isotani et al. 2009; Isotani e Mizoguchi 2007] com a proposta de auxiliar na tarefa de formação de grupos, no planejamento instrucional de atividades e nas estratégias para promover a interação entre os alunos. São exemplos de teorias ${ }^{2}$ de aprendizagem colaborativa, Anchored Instruction [CTGV 1992] e Cognitive Apprenticeship [Collins 1991].

No design para formação de grupos em ambientes CSCL, Inaba e Mizoguchi (2004) e Isotani et al. (2009) enfatizam a importância da atribuição adequada de papéis entre os participantes para que, baseado na teoria de aprendizagem definida, os envolvidos assumam (ou adotem) determinado comportamento para alcançar os benefícios de aprendizagem previstos. Vale ressaltar que os autores utilizam um modelo simplificado, com no máximo dois papéis para cada teoria de aprendizagem. Como resultado, o trabalho de Inaba e Mizoguchi (2004) apresenta nove tipos de comportamento relacionados à treze tipos de papéis. Um exemplo dessa relação, baseado na teoria de aprendizagem Anchored Instruction (CTGV, 1992), pode ser visualizado na Tabela 1.

Tabela 1. Exemplo de papéis e seus elementos para a teoria de aprendizagem Anchored Instruction (Fonte: Inaba e Mizoguchi 2004).

\begin{tabular}{|l|l|l|l|}
\hline \multicolumn{1}{|c|}{ Papel } & Comportamento/Função & \multicolumn{1}{c|}{ Condição Cognitiva } & Benefícios de Aprendizagem \\
\hline $\begin{array}{l}\text { Instrutor } \\
\text { (anchored } \\
\text { instructor })\end{array}$ & $\begin{array}{l}\text { Aconselhamento } \\
\text { (advising): diagnosticar } \\
\text { problemas e } \\
\text { aconselhar/orientar os } \\
\text { colegas. }\end{array}$ & $\begin{array}{l}\text { (1) Ter domínio do conteúdo a } \\
\text { ser abordado. } \\
\text { (2) Conhecer estratégias para } \\
\text { diagnosticar problemas. } \\
\text { (3) Não ter experiência em } \\
\text { diagnosticar problemas na } \\
\text { prática. }\end{array}$ & $\begin{array}{l}\text { Aquisição de conhecimento } \\
\text { sobre o conteúdo abordado. } \\
\text { (acquisition of tunning) }\end{array}$ \\
$\begin{array}{l}\text { Desenvolvimento de } \\
\text { habilidade cognitiva. } \\
\text { (development of associative) }\end{array}$ \\
\hline $\begin{array}{l}\text { Instruído } \\
\text { (problem } \\
\text { holder) }\end{array}$ & $\begin{array}{l}\text { Apresentação (presenting): } \\
\text { explicar aos colegas algum } \\
\text { conteúdo com suas } \\
\text { próprias palavras. }\end{array}$ & $\begin{array}{l}\text { (1) Ter um problema. } \\
\text { (2) Conhecer os conteúdos } \\
\text { relacionados ao problema. }\end{array}$ & $\begin{array}{l}\text { Aquisição de conhecimento } \\
\text { sobre o conteúdo abordado. } \\
\text { (acquisition of tunning) }\end{array}$ \\
\hline
\end{tabular}

Conforme mostrado na Tabela 1, cada papel possui um comportamento previamente estabelecido. Por exemplo, ao desempenhar o papel de Instrutor (anchored instructor), o aluno adotará o comportamento de aconselhamento (advising) com a função de diagnosticar problemas e aconselhar/orientar os colegas. Por outro lado, se o aluno desempenhar o papel de Instruído (problem holder), seu comportamento será de apresentação (presenting), ou seja, explicar aos colegas algum conteúdo com suas próprias palavras.

Além do comportamento para alcançar os benefícios de aprendizagem previstos (Tabela 1), Inaba e Mizoguchi (2004) discutem a importância de definir os requisitos (condições necessárias e condições desejadas) para que o aluno possa desempenhar determinado papel. As condições necessárias são aquelas essenciais para que o aluno

\footnotetext{
2 Os nomes das teorias de aprendizagem são mantidos em inglês, conforme referenciados em Inaba e Mizoguchi (2004).
} 
possa desempenhar o papel, ou seja, se o aluno não atender a essas condições não será possível assumir o papel estabelecido. As condições desejadas definem os requisitos que o aluno deve satisfazer para assegurar os benefícios de aprendizagem previstos. Por exemplo, de acordo com a Tabela 1, o aluno que desempenhar o papel de Instrutor terá como condições necessárias: (1) ter conhecimento sobre o conteúdo que será abordado, (2) conhecer estratégias para diagnosticar problemas; e como condição desejada (3) não ter experiência em diagnosticar problemas na prática. Nesse exemplo, as condições necessárias 1 e 2 são obrigatórias para o papel assumido pelo aluno e a condição 3, caso seja atendida, garantirá os ganhos de aprendizagem ao aluno que, após desempenhar o papel de Instrutor, terá, por exemplo, desenvolvido sua habilidade cognitiva no diagnóstico de problemas.

No intuito de representar computacionalmente os cenários de aprendizagem colaborativa, Isotani et al. (2009) apresentam um modelo de ontologias ${ }^{3}$ (framework) baseado em teorias de aprendizagem para melhorar o planejamento instrucional e a formação de grupos em cenários CSCL. Apesar da importante contribuição dos trabalhos de Inaba e Mizoguchi (2004) e Isotani et al. (2009) em estabelecer a relação e representação computacional (por meio de ontologias) entre os papéis e as teorias de aprendizagem, observou-se que os estados afetivos, elementos que também influenciam na aprendizagem [Leite e Tassoni 2002], não foram considerados nessa modelagem. Nesse sentido, com o intuito de contribuir para a ampliação das propostas, as próximas seções são dedicadas à definição e caracterização dos estados afetivos (Seção 3.2), bem como em estabelecer a relação entre o estado afetivo "traço de personalidade" e os papéis assumidos pelos aprendizes (Seção 4). Essa relação dá origem a novos papéis, tratados nesse artigo como "papéis afetivos".

\subsection{Estados Afetivos e suas Características/Comportamentos}

De acordo com Scherer (2000), os estados afetivos são classificados em 5 tipos: emoções (emotion), estados de ânimo (mood), posturas interpessoais (interpersonal instance), atitudes (attitudes) e traços de personalidade (personality trait). Este trabalho possui interesse particular nos traços de personalidade, definidos como estados afetivos relativamente estáveis, previsíveis e característicos de uma pessoa [Scherer 2000], e na relação desses traços com os papéis (discutida na Seção 4) que os alunos podem assumir nas diversas teorias de aprendizagem. Para que seja possível estabelecer essa relação, é preciso primeiramente selecionar os traços de personalidade, representados na Tabela 2 pelos $\operatorname{traços}^{4}$ de extroversão e introversão, bem como suas características e comportamentos. Vale ressaltar que esses traços de personalidade foram retirados de

\footnotetext{
${ }^{3}$ Uma ontologia é caracterizada como um sistema de conceitos fundamentais para representar um domínio de conhecimento específico [Gruber 1993], que pode ser aplicada em sistemas computacionais inteligentes [Isotani et al. 2008].

${ }^{4}$ Devido a limitação de espaço, nesse artigo serão considerados apenas os traços de personalidade de extroversão e introversão, mas a presente proposta se aplica aos demais traços dos questionários de personalidade Big Five e MBTI.
} 
questionários psicológicos consolidados na literatura como Big Five [Barrick e Mount 1991] e MBTI [Myers et al. 1998].

Tabela 2. Características e comportamentos dos traços de personalidade Extroversão e Introversão.

\begin{tabular}{|l|l|l|l|}
\hline \multicolumn{1}{|c|}{$\begin{array}{c}\text { Traço de } \\
\text { personalidade }\end{array}$} & \multicolumn{1}{|c|}{ Características } & \multicolumn{1}{|c|}{ Comportamentos } & Referência \\
\hline Extroversão & $\begin{array}{l}\text { Impulsividade; despreocupação; } \\
\text { agressividade; otimismo; espontaneidade; } \\
\text { autoconfiança; amigabilidade; assertividade; } \\
\text { entusiasmo; audácia. }\end{array}$ & $\begin{array}{l}\text { Sociabilidade; liderança; } \\
\text { interação; atividade; vivacidade; } \\
\text { desinibição; dispersão. }\end{array}$ & $\begin{array}{l}\text { Big Five } \\
\text { MBTI }\end{array}$ \\
\hline Introversão & $\begin{array}{l}\text { Reflexão; inibição; discrição; timidez; } \\
\text { responsabilidade. }\end{array}$ & Rigidez; introspecção. & MBTI \\
\hline
\end{tabular}

De acordo com a Tabela 2, cada traço de personalidade possui características próprias que dão origem a determinados comportamentos. Esses elementos serão relacionados com os papéis que os alunos podem desempenhar (Seção 4) em um cenário CSCL, para estabelecer os novos requisitos (condições necessárias e desejadas) e comportamentos para os novos papéis (papéis afetivos) que surgirem.

\section{Resultados: Modelagem dos "Papéis Afetivos" em CSCL}

Dado que o objetivo deste trabalho consiste em estabelecer a relação entre os estados afetivos e os papéis que os aprendizes podem assumir nas diversas teorias de aprendizagem, nesta seção, essa relação é ilustrada e exemplificada com base nos traços de personalidade (TP) extroversão e introversão, apresentados na Seção 3.2; e nos comportamentos e requisitos dos papéis (P) da teoria de aprendizagem Anchored Instruction, discutidos na Seção 3.1. Como resultado dessa relação (TP x P), foram criados novos papéis, denominados "papéis afetivos", e definidos os seus comportamentos e requisitos (condições necessárias e desejadas), conforme mostra a Tabela 3. Vale ressaltar que, com a definição dos "papéis afetivos", e os comportamentos que esses papéis podem assumir, torna-se necessário determinar as estratégias para assegurar os benefícios previstos por Inaba e Mizoguchi (2004) para cada teoria de aprendizagem.

Nesse sentido, para delinear o comportamento do aluno ao desempenhar um papel afetivo (Tabela 3), foram analisadas as características/comportamentos dos TPs introversão e extroversão (Tabela 2) em relação aos comportamentos/funções dos papéis (Tabela 1). Por exemplo, de acordo com a Tabela 2, um indivíduo com TP introversão tem como comportamento afetivo a reflexão, inibição, timidez, discrição, etc. O papel de Instrutor, quando atribuído a esse indivíduo, dará origem ao papel afetivo Instrutor Introvertido (introverted anchored instructor) (Tabela 3). O comportamento esperado ao desempenhar esse papel é ter "habilidade para diagnosticar problemas", devido à característica afetiva de reflexão antes de agir, e "dificuldade para orientar/aconselhar os colegas", que está relacionado à timidez, inibição. Dessa forma, para assegurar os benefícios de aprendizagem desse indivíduo, sugere-se "criar situações que promovam a 
interação assíncrona" (Tabela 3) que pode ser realizada, por exemplo, por meio de ferramentas como os fóruns de discussão.

Outro exemplo é a atribuição do papel Instruído a um aluno com TP extrovertido, que dará origem ao papel afetivo Instruído Extrovertido (extroverted problem holder) (Tabela 3). O comportamento esperado nesse caso é de "confusão para explicar algum conhecimento aprendido", devido à característica de dispersão. Para assegurar os benefícios de aprendizagem, recomenda-se "propor estratégias que favoreçam a organização do conteúdo (ou conhecimento)", que pode ser feita, por exemplo, por meio de esquemas como mapas conceituais.

A Tabela 3 apresenta também as condições necessárias, sinalizadas com o sinal de asterisco (*), e condições desejadas, sinalizadas com o sinal de hífen (-), para que um aprendiz possa assumir o papel afetivo de Instrutor/Instruído Extrovertido/Introvertido, por exemplo. Lembrando que as condições cognitivas apresentadas na Tabela 3 foram herdadas da Tabela 1.

Em relação às condições afetivas têm-se, por exemplo, que para que um aluno desempenhe o papel de Instruído Introvertido a condição necessária é que esse aluno seja introvertido e a condição desejada é que ele não seja aberto à interação. E, para que os benefícios de aprendizagem sejam assegurados, sugere-se a criação de situações que promovam a interação entre os alunos e que possibilite ao aprendiz explicar, por exemplo, algo que seja do seu conhecimento e/ou apresentar questionamentos. $\mathrm{O}$ mesmo raciocínio pode ser feito para os demais papéis afetivos.

Tabela 3. Exemplo de papéis afetivos e suas características.

\begin{tabular}{|c|c|c|c|c|}
\hline Papel afetivo & Condição Cognitiva/Afetiva & Comportamento & $\begin{array}{l}\text { Benefícios de } \\
\text { Aprendizagem }\end{array}$ & $\begin{array}{c}\text { COMO } \\
\text { assegurar os } \\
\text { Benefícios de } \\
\text { Aprendizagem? }\end{array}$ \\
\hline \begin{tabular}{|l|}
\multicolumn{1}{|c|}{ Instrutor } \\
Extrovertido \\
(extroverted \\
anchored \\
instructor)
\end{tabular} & $\begin{array}{l}\text { * Ter domínio do conteúdo a ser } \\
\text { abordado (cognitivo). } \\
\text { * Conhecer estratégias para } \\
\text { diagnosticar problemas (cognitivo). } \\
\text { - Não ter experiência em diagnosticar } \\
\text { problemas na prática (cognitivo). } \\
\text { * Ter TP de extroversão (afetivo). } \\
\text { - Não ser reflexivo (afetivo). }\end{array}$ & $\begin{array}{l}\text { Confusão para } \\
\text { diagnosticar } \\
\text { problemas e } \\
\text { facilidade para } \\
\text { orientar, } \\
\text { aconselhar os } \\
\text { colegas. }\end{array}$ & \multirow{2}{*}{$\begin{array}{l}\text { Aquisição de } \\
\text { conhecimento } \\
\text { sobre o conteúdo } \\
\text { abordado } \\
\text { (acquisition of } \\
\text { tunning) } \\
\text { Desenvolvimento } \\
\text { de habilidade } \\
\text { cognitiva } \\
\text { (development of } \\
\text { associative) }\end{array}$} & $\begin{array}{l}\text { Controlar } \\
\text { situação por } \\
\text { meio de } \\
\text { atividades pré- } \\
\text { planejadas. }\end{array}$ \\
\hline \begin{tabular}{|} 
Instrutor \\
Introvertido \\
(introverted \\
anchored \\
instructor)
\end{tabular} & $\begin{array}{l}\text { *Ter domínio do conteúdo a ser } \\
\text { abordado (cognitivo). } \\
\text { * Conhecer estratégias para } \\
\text { diagnosticar problemas (cognitivo). } \\
\text { - Não ter experiência em diagnosticar } \\
\text { problemas na prática (cognitivo). } \\
\text { *Ter TP de introversão (afetivo). } \\
\text { - Não ser aberto à interação (afetivo). }\end{array}$ & $\begin{array}{l}\text { Habilidade para } \\
\text { diagnosticar } \\
\text { problemas e } \\
\text { dificuldade para } \\
\text { orientar/aconselhar } \\
\text { os colegas. }\end{array}$ & & $\begin{array}{c}\text { Criar situações } \\
\text { que promovam a } \\
\text { interação } \\
\text { assíncrona. }\end{array}$ \\
\hline \begin{tabular}{|c|} 
Instruído \\
Extrovertido
\end{tabular} & $\begin{array}{l}\text { * Ter um problema. } \\
\text { - Conhecer os conteúdos relacionados }\end{array}$ & $\begin{array}{l}\text { Confusão para } \\
\text { explicar algum }\end{array}$ & $\begin{array}{l}\text { Aquisição de } \\
\text { conhecimento }\end{array}$ & $\begin{array}{c}\text { Propor } \\
\text { estratégias que }\end{array}$ \\
\hline
\end{tabular}


CBIE-LACLO 2015

Anais do XXVI Simpósio Brasileiro de Informática na Educação (SBIE 2015)

\begin{tabular}{|c|c|c|c|c|}
\hline $\begin{array}{l}\text { (extroverted } \\
\text { problem } \\
\text { holder) }\end{array}$ & $\begin{array}{l}\text { ao problema. } \\
\text { * Ter TP de extroversão (afetivo). } \\
\text { - Não ser reflexivo (afetivo). }\end{array}$ & $\begin{array}{l}\text { conhecimento } \\
\text { aprendido. }\end{array}$ & \multirow[t]{2}{*}{$\begin{array}{l}\text { sobre o conteúdo } \\
\text { abordado } \\
\text { (acquisition of } \\
\text { tunning) }\end{array}$} & $\begin{array}{l}\text { favoreçam a } \\
\text { organização do } \\
\text { conteúdo. }\end{array}$ \\
\hline $\begin{array}{l}\text { Instruído } \\
\text { Introvertido } \\
\text { (introverted } \\
\text { problem } \\
\text { holder) }\end{array}$ & $\begin{array}{l}* \text { Ter um problema. } \\
\text { - Conhecer os conteúdos relacionados } \\
\text { ao problema. } \\
* \text { Ter TP de introversão (afetivo). } \\
\text { - Não ser aberto à interação (afetivo). }\end{array}$ & $\begin{array}{l}\text { Dificuldade para } \\
\text { explicitar algo que } \\
\text { seja do seu } \\
\text { conhecimento. }\end{array}$ & & $\begin{array}{l}\text { Criar situações } \\
\text { que promovam a } \\
\text { interação } \\
\text { assíncrona. }\end{array}$ \\
\hline
\end{tabular}

\subsection{Extensão de uma Ontologia para CSCL utilizando Estados Afetivos}

Com o intuito de considerar os estados afetivos na formação de grupos de alta performance, e melhorar o planejamento instrucional de cenários CSCL, este trabalho além de definir os novos papéis afetivos, propõe estender a estrutura ontológica de papéis (CL Role) proposta na ontologia colaborativa de Isotani et al. (2009). A Figura 1 (a) mostra a nova estrutura ontológica de papéis (CL role) em que os traços de personalidade são definidos como condições necessárias (necessary condition) e desejáveis (desired condition), sinalizados pelas linhas tracejadas.

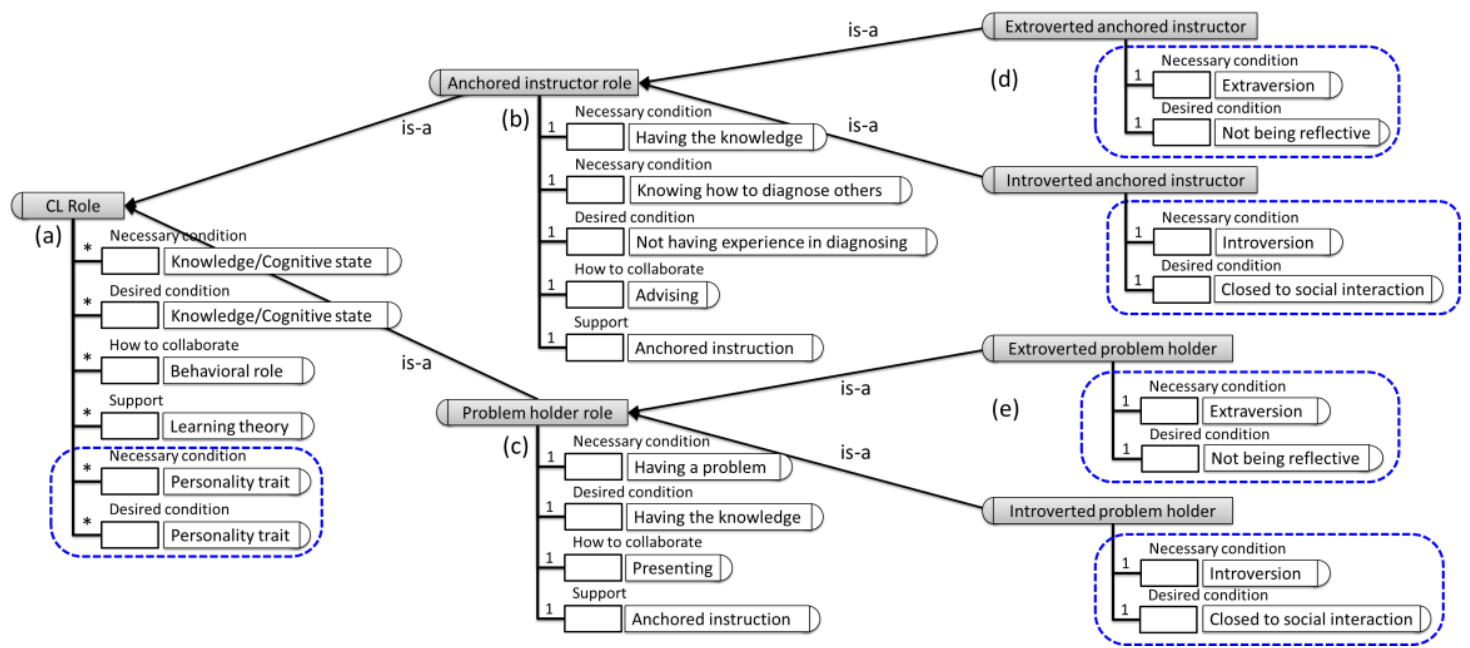

Figura 1 - Estrutura ontológica para representar papéis nos cenários de aprendizagem colaborativa, e exemplos de papéis/papéis afetivos.

Empregando a nova estrutura mostrada na Figura 1(a) é possível representar especializações dos papéis definidos para cenários de aprendizagem colaborativa. A Figura 1(d) mostra como exemplo a definição dos papéis de Instrutor Extrovertido (Extroverted anchored instructor) e Instrutor Introvertido (Introverted anchored instructor) que são especializações do papel instrutor (Anchored instructor role) mostrado na Figura 1(b). Como exemplo de especializações do papel instruído (Problem holder role) mostrado na Figura 1(c), a Figura 1(e) mostra os papéis de Instruído Extrovertido (Extroverted problem holder) e Instruído Introvertido (Introverted problem holder). De acordo com esses exemplos, é necessário que os estudantes com papéis de Instrutor Extrovertido (Extroverted anchored instructor) e Instruído Extrovertido (Extroverted problem holder) tenham traço de personalidade extrovertido (extraversion), sendo assim definidos como condição necessária (necessary condition), 
enquanto a condição desejada (desired condition) é que não sejam reflexivos (not being reflective). Da mesma forma, o traço de personalidade introvertido (introversion) é definido como condição necessária (necessary condition) e não possuir abertura à interação (closed to social interaction) é definido como condição desejada (desired condition) para desempenhar os papéis de Instrutor Introvertido (Introversion anchored instructor) e Instruído Introvertido (Introversion problem holder).

\section{Conclusão}

A formação de grupos de alta performance é apontada como um dos desafios da CSCL no que se refere a assegurar a sinergia entre os seus membros, para que os benefícios educacionais sejam alcançados de forma plena. No intuito de promover essa sinergia, este trabalho apresentou uma proposta de relação entre os estados afetivos do tipo traço de personalidade (e.g., extroversão, introversão) e os papéis que os alunos podem desempenhar (e.g., instrutor, instruído) nas diversas teorias de aprendizagem colaborativa (e.g., Anchored Instruction). Como resultados, foram definidos novos papéis ("papéis afetivos"), com novos comportamentos e requisitos (condições necessárias e desejadas), modelados como uma extensão à estrutura ontológica de papéis desenvolvida por Isotani et al. (2009), para o contexto da CSCL.

Como trabalhos futuros vislumbra-se o desenvolvimento de cenários CSCL que utilizem a ontologia apresentada para auxiliar na formação de grupos de alta performance. Além disso, sugere-se a ampliação dos estados afetivos para que as "emoções" e "estados de ânimo" também sejam considerados nos papéis afetivos.

\section{Agradecimentos}

Os autores gostariam de agradecer apoio financeiro do CNPq e da CAPES.

\section{Referências}

Barrick, M. R. and Mount, M. K. (1991). "The Big Five personality dimensions and job performance: a meta-analysis", Personnel Psychology, 44: 1-26.

CTGV - Cognition and Technology Group at Vanderbilt (1992). "Anchored instruction in science education", In: R. Duschl \& R. Hamilton (Eds.), Philosophy of science, cognitive psychology, and educational theory and practice. Albany, NY: SUNY Press, p. 244-273.

Collins, A. (1991). "Cognitive apprenticeship and instructional technology". Educational values and cognitive instruction: Implications for reform. Hillsdale, N.J.: LEA.

Flor, J. M. and Finger, S. (2006). "The Effect of Individual and Group Characteristics on Remote Collaboration", In Proceeding of the 36th Annual Conference Frontiers in Education, p. 23-28.

Dillenbourg, P. (2002). "Over-scripting CSCL: The risks of blending collaborative learning with instructional design", In: P. A. Kirschner (Ed.), Three worlds of CSCL. Can we support CSCL? (p. 61-91). Heerlen, NL: Open Universiteit Nederland.

Gruber, T. R. (1993). "A Translation Approach to Portable Ontology Specification", Knowledge Acquisition, 5: 199-220. 
Hsu, J. -L.,Chou, H.-W., Hwang, W.-Y. and Chou. S.-B. (2008). "A Two-Dimension Process in Explaining Learners' Collaborative", Behaviors in CSCL. Educational Technology \& Society.

Inaba, A. and Mizoguchi, R. (2004). "Learner's Role and Predictable Educational Benefits in Collaborative Learning", In Proceeding of the International Conference on Intelligent Tutoring Systems, LNCS 3220, Springer-Verlag, p. 285-294.

Isotani, S., Inaba, A., Ikeda, M. and Mizoguchi, R. (2009). "An ontology engineering approach to the realization of theory-driven group formation", International Journal of Computer-Supported Collaborative Learning, 4(4): 445-478.

Isotani, S., Isotani, N. and Isotani, S. (2008). "Ontologias e Web Semântica no Suporte ao Ensino Colaborativo em Salas de Aula Presenciais", Anais do Simpósio Brasileiro de Informática na Educação, 1(1): 521-531.

Isotani, S. and Mizoguchi, R. (2007). "Planejamento e Análise de Sessões Colaborativas Utilizando Teorias de Aprendizagem e Ontologias", Revista Brasileira de Informática na Educação, 15(2): 45-56.

Leite, S. A. S., Tassoni, E. C. M. (2002). "A afetividade em sala de aula: as condições de ensino e a mediação do professor", In: Azzi, R. G., Sadalla, A. M. F. A. (Org.). Psicologia e formação docente: desafios e conversas. São Paulo: Casa do Psicólogo.

Lopes Filho, J. A. B., Quarto, C. C. and França, R. M. (2010). "Uma interface Web em conjunto com algoritmo de clustering no auxílio à formação de grupos colaborativos sócio-afetivos”, XXI Simpósio Brasileiro de Informática na Educação (SBIE 2010).

Mizoguchi, R. (2004). "Tutorial on ontological engineering - part 3: Advanced course of ontological engineering", New Generation Computing, 22(2): 198-220.

Myers, I. B., McCaulley, M. H., Quenk, N. L. and Hammer, A. L. (1998). "MBTI Manual: A Guide to the Development and Use of the Myers-Briggs Type Indicator", Palo Alto, CA: Consulting Psychologists Press, third Edition.

Quarto, C. C., Labidi, S., Jaques, P. and Schivitz, I. M. (2006). "Considerando os fatores sócio-afetivos personalidade e liderança em ambientes de ensino-aprendizagem colaborativos assistidos por computador". Simpósio Brasileiro de Informática na Educação (SBIE 2006), Brasília, DF, 17: 13-22.

Roschelle, J. and Teasley, S. D. (1995). "The Construction of Shared Knowledge", In Collaborative Problem Solving Computer Supported Collaborative Learning. C. E. O’Malley. NY, Springer- Verlag: p. 69-97.

Scherer, K. R. (2000) "Psychological models of emotion", In: J. Borod (Ed.), The neuropsychology of emotion. Oxford University Press, 37: 137-162.

Silva, F. E. O., Motta, C.L.R. and Santoro, F.M. (2010). "Team Composer: Assembling groups through social matching", In Proceeding of the International Conference on Computer Supported Cooperative Work in Design, p. 128-133.

Tchounikine, P., Rummel, N. and McLaren, B. M. (2010). "Computer Supported Collaborative Learning and Intelligent Tutoring Systems", In Advances in Intelligent Tutoring Systems, Edited by R. Nkambo, J. Bourdeau, \& R. Mizoguchi, chapter 22, p. 447-463. Springer.

Wang, D. -Y., Lin, S. S. J. and Sun, C. -T. (2007). "DIANA: A Computer-Supported Heterogeneous Grouping System for Teachers to Conduct Successful Small Learning Groups", Computers in Human Behavior, 23: 1997-2010. 